\title{
Weiterbildungsförderung bei regionaler Unterversorgung verlängert
}

\begin{abstract}
- Kassenärztliche Bundesvereinigung (KBV) und Kassen haben sich auf eine Verlängerung der Zusatzvereinbarung zur finanziellen Förderung der Weiterbildung in der Allgemeinmedizin bei lokaler Unterversorgung bis zum 31. Dezember 2013 geeinigt. Die Vereinbarung war bis zum 31. Dezember 2012 befristet und sieht jetzt zusätzlich für eine hausärztliche Weiterbildung auch in einem nicht unterversorgten Planungsbereich einen erhöhten Förderbetrag vor, wenn der zuständige Landesausschuss einen zusätzlichen lokalen Versorgungsbedarf festgestellt hat. Vor Abschluss der Vereinbarung war dies nur in (drohend) unterversorgten Bereichen möglich.
\end{abstract}

\section{MMW Kommentar}

Die regionale Umsetzung dieses Beschlusses hängt allerdings von den Änderungen in der Bedarfsplanungsrichtlinie ab. Wenn diese Auswirkungen auf den zusätzlichen lokalen Versorgungsbedarf haben sollte (\$34a der derzeit gültigen Bedarfsplanungsrichtlinie des Gemeinsamen Bundesausschusses), tritt die Regelung bereits vor Ablauf des 31. Dezember 2013 außer Kraft. Der Gemeinsame Bundesausschuss (GBA) hat in seiner Sitzung vom 20. Dezember 2012 die neue Bedarfsplanungs-Richtlinie beschlossen. Den KVen verbleiben zur Umsetzung der Richtlinie auf Landesebene nun sechs Monate Zeit zur Erstellung des neuen Bedarfsplans.

\section{„Verweilen beim Patienten“}

— Die Leistung nach EBM-Ziffer 01440 ist „für das Verweilen außerhalb der Praxis ohne Erbringung weiterer berechnungsfähiger Gebührenordnungspositionen, wegen der Erkrankung erforderlich, je vollendete 30 Minuten" berechnungsfähig. Mögliche Fälle, bei denen diese Leistung denk- bar ist, wären ein Hausbesuch bei einem älteren Patienten oder bei einem $\mathrm{Pa}$ tienten, bei dem auf das Einsetzen der Wirkung eines applizierten Medikaments gewartet werden muss oder wenn man bei einem Patienten auf das Eintreffen eines Krankentransports wartet.

\section{MMW Kommentar}

Die Leistung nach Ziffer 01440 kann aufgrund der Leistungslegende aber nur dann abgerechnet werden, wenn innerhalb der Verweilzeit keine berechnungsfähigen Leistungen erbracht wurden. Der für die Erbringung von Leistungen erforderliche Zeitbedarf, beispielsweise bei einer Infusion, ist mit der Bewertung für die entsprechenden Leistungen abgegolten. Warten man bei dem oben beschriebenen Hausbesuch nach Beendigung der Infusion 30 Minuten oder länger, ist die Nr. 01440 EBM zusätzlich zum Besuch berechnungsfähig. Aufgrund der Leistungslegende zur Nr. 01440 EBM sowie der Allgemeinen Bestimmungen ist die Verweilgebühr im Zusammenhang mit der Erbringung von Leistungen in der Praxis hingegen nicht berechnungsfähig.

\section{Neu: Heilmittel- Verordnung}

— Seit Jahresbeginn gilt erstmals bundesweit ein Katalog für Praxisbesonderheiten bei Heilmitteln. Das bisherige Verfahren der Meldung von Praxisbesonderheiten wurde deshalb abgeschafft und die bisherigen Symbolnummern gelten nicht mehr. Will man eine Praxisbesonderheit bei der Heilmittelverordnung melden, muss jetzt die jeweilige ICD-10-Kodierung auf dem Heilmittel-Verordnungsformular (Muster $13,14,18$ ) handschriftlich eintragen werden. Bei einer solchen Kennzeichnung wird das Heilmittel-Budget der Praxis nicht belastet!

\section{MMW Kommentar}

Leider fehlt bislang auf dem Heilmittel-Verordnungsformular dieses Feld für die ICD-10Kodierung, da sich die Neuregelung noch im Unterschriftsverfahren befindet. Es empfiehlt sich deshalb, die Kodierung an geeigneter Stelle in dem Diagnosefeld des alten Formulars einzutragen, bis dieses dem neuen Verfahren angepasst wurde. Die Meldung von Praxisbesonderheiten soll dann auch durch eine aktualisierte Praxissoftware unterstützt werden. Wird eine Heilmittel-Verordnung in Verbindung mit dem entsprechenden ICD10-Code der Praxisbesonderheit ausgestellt, erledigt die Praxis-Software die Meldung der Praxisbesonderheit automatisch.

Eine solche Software wird allerdings voraussichtlich erst ab dem 2. Quartal 2013 zur Verfügung stehen. Beachtenswert ist auch, dass es eine Liste der per ICD-10 gesteuerten Möglichkeiten einer Genehmigung zum langfristigen Heilmittelbedarf gibt, den der Patient bei seiner Kasse beantragen muss. Diese Fälle fallen bei entsprechender Genehmigung ebenfalls aus dem Heilmittel-Budget heraus. 